\title{
An Optimum Design Method of Pneu-Net Actuators for Trajectory Matching Utilizing a Bending Model and GA
}

\author{
Yilin Sun $\mathbb{D}^{1},{ }^{1,2}$ Qiuju Zhang $\mathbb{D}^{1,2}$ Xiaoyan Chen, ${ }^{1,2}$ and Haiwei Chen ${ }^{1,2}$ \\ ${ }^{1}$ School of Mechanical Engineering, Jiangnan University, Wuxi 214122, China \\ ${ }^{2}$ Jiangsu Key Laboratory of Advanced Food Manufacturing Equipment and Technology, Jiangnan University, \\ Wuxi 214122, China \\ Correspondence should be addressed to Yilin Sun; 15261596813@163.com and Qiuju Zhang; qjzhang@jiangnan.edu.cn
}

Received 24 May 2019; Accepted 9 August 2019; Published 14 October 2019

Academic Editor: Arkadiusz Zak

Copyright (C) 2019 Yilin Sun et al. This is an open access article distributed under the Creative Commons Attribution License, which permits unrestricted use, distribution, and reproduction in any medium, provided the original work is properly cited.

\begin{abstract}
This study addresses the problem in designing pneu-net actuators to achieve specific target trajectories for particular applications. The finite element analysis (FEA) method is used to study the sensitivity of 9 design parameters to bending deformation of actuators, and the results of FEA demonstrate that the width of the chamber and the width of the middle layer have great influence on the bending performance. Besides, the relations between the bending angles of actuators with different width parameters and the pressure are always highly linear. Using FEA and the cubic polynomial fitting algorithm, a unified bending model of the pneunet actuator is established with three crucial design parameters (i.e., segment length, chamber width, and middle-layer width). An optimum design method based on the bending model and genetic algorithm (GA) is put forward to automatically adjust these three crucial parameters to realise trajectory matching. The method is developed to be an effective solution for the problem in matching the target trajectory with the experimental results achieved on an actuator designed by imitating a human index finger. Furthermore, the results of the experiment also verify that the actuator can achieve stable grasping, and the proposed method has an important directive to design pneu-net actuators for their application in the fields of robotics, rescue, and detection.
\end{abstract}

\section{Introduction}

Soft actuators are widely fabricated by using compliant materials (e.g., polymers [1], elastomers [2], and hydrogels [3-5]) which result in a vast potential of soft actuators to handle fragile objects, resist mechanical insults (e.g., blunt impacts and compressive forces), and realise complex motions [6-8]. Pneumatically powered actuators aim to use new design strategies so that they can rapidly provide complex motions with simple inputs, thereby inspiring a new wave of research activities [9-11]. Recently, the field of soft pneumatic actuators (SPAs) has achieved a remarkable progress with the development of biomimetic materials science [12-14] and advanced manufacturing processes [15].

Fibre-reinforced and pneumatic network (pneu-net) actuators are the two most popular categories among the abundant designs of SPAs $[7,8,16]$. A fibre-reinforced actuator is mainly composed of an elastomer body with a large chamber in the centre, a strain-limiting layer at the bottom, and helically arranged fibres on the surface; these fibres protect the actuator from excessive deformation [17]. Fibre-reinforced actuators can achieve extending, twisting, expanding, and bending motions and follow a designed kinematic trajectory by changing the components and tuning oblique angle and fibre length $[7,18]$. A pneu-net actuator is composed of an inextensible bottom layer and an elastomer body with a network of small chambers inside; these small chambers lead to the deformation of actuators when inflated $[6,8,17]$. A pneu-net actuator can also realise bending and twisting motions by altering the chamber angle [8].

The field of SPAs has achieved a remarkable progress with the development of many soft grippers $[19,20]$, locomotion robots [21, 22], bionic robots [23-25], and biomedical devices for rehabilitation [26-28]. Simultaneously, the methods used for efficiently designing actuators for particular functions are essential to studying SPAs [29]. On 
this basis, many research groups have focused on the theoretical framework for modelling SPAs, although developing realistic and tractable models is challenging $[9,11]$. Therefore, designing pneu-net actuators to perform a particular trajectory remains a challenge.

In this study, an efficient strategy is proposed to determine the optimal design parameters for a pneu-net actuator that can match a desired trajectory upon pressurisation. Numerous geometry models with 9 different design parameters are designed to investigate the effect of design parameters on the bending performance of pneu-net actuators in Section 2. Using the technology of FEA, we can see that the effects of these two parameters (i.e., the chamber width and the middle-layer width) on the performance of the actuator are more sensitive than those of the other parameters. Section 3 proposes a GAbased optimum design method of actuators for realising trajectory matching. In Section 4, based on the proposed method, an actuator is designed to match the trajectory of a human index finger. Section 5 presents some conclusions of this work.

\section{FEA for Pneu-Net Actuator}

Pneu-net actuators have three typical geometries: (a), (b), and (c) $[17,20]$, as illustrated in Figure 1. Considerable deformations and stresses are excited on the top wall of geometry (a) and geometry (b) when pressurised, thereby possibly inducing failure when the top walls are pierced or the air pressure is largely increased. The main deformation zone of geometry (c) is the inside walls that may contact each other when the chambers are inflated. This form of bending motion can protect the walls from excessive deformation and thus induce the development of reliability over geometry (a) and geometry (b). Thus, geometry (c) is selected for intensive research in this work.

In theory, different combinations of geometrical parameters can produce actuators with different performance, which are also called design parameters. For distinguishing which design parameters have a remarkable effect on the performance of the actuator, a set of pneu-net actuators is designed with different design parameters, and each of them has one specific parameter that is different from that of the reference actuator. The length, width, and height of the actuators are fixed, that is, 85,26 , and $20 \mathrm{~mm}$, respectively. The geometric structure of the reference actuator is depicted in detail in Figure 2, and the corresponding design parameters are listed in Table 1.

The FEA has been widely applied as a design tool $[7,17]$, and its practicality has been confirmed in determining the optimal design parameters. Therefore, the FEA is used to analyse the influence of design parameters on the performance of the actuator. The main performance index of the actuator is the bending ability, that is, the bending angle of the actuator. The geometry models of actuators are designed in SolidWorks 2014 (Dassault Systems) and then imported into ABAQUS CAE 6.14-3 (Dassault Systems) for FEA simulations. After loading the geometry models, the end of the actuator is constrained, and pressure is applied to the walls. The material behavior is defined as hyperelastic, and the material type is isotropic. Uniaxial tensile tests are executed according to the standard ASTM D638 to determine the constitutive model parameters of the actuator's material (i.e., Dragon Skin 30). After evaluating other constitutive models, the Yeoh model is considered to be the best constitutive relation for the applications in this paper. The stress-strain curve is exhibited in Figure 3.

FEA-simulated images of the bending motion of actuators are demonstrated in Figure 4. The FEA result of the reference actuator is shown in Figure 4(a). In Figure 4, it can be seen that the bending angle of the actuator $\mathrm{f}$ (with the middle-layer width parameter $w_{4}=8 \mathrm{~mm}$ ) has remarkably increased over that of the reference actuator under the same pressure $(5 \mathrm{kPa})$. Moreover, the middle layer of each of the eight actuators is the stress concentration region. Hence, the middle-layer width parameter has a remarkable effect on the bending performance of actuators. In comparison with other actuators, the bending angle of the actuator $b$ (with the chamber width parameter $w_{2}=20 \mathrm{~mm}$ ) is also slightly increased. Thus, subsequent research is conducted on the effects of the two width parameters $\left(w_{2}\right.$ and $\left.w_{4}\right)$ on the bending performance of actuators.

\section{Optimization Strategy of Design Parameters}

3.1. Sensitivity Analysis of the Two Width Parameters. To analyse the sensitivity of bending angle to the variation of the chamber width parameter (i.e., $w_{2}$ ) under the same pressure and different pressures, a series of actuators (i.e., M2 - 1, $\mathrm{M} 2-2, \mathrm{M} 2-3, \mathrm{M} 2-4, \mathrm{M} 2-5, \mathrm{M} 2-6, \mathrm{M} 2-7$, and M2-8) with different values of the width parameter $w_{2}\left(w_{2-i}=18.4\right.$, $18.8,19.2,19.6,20,20.4,20.8$, and $21.2 \mathrm{~mm}$ ) are designed, respectively, and the FEA simulations are conducted.

The parameter $\theta$ is used to represent the bending angle of the reference actuator, $\theta_{i}$ represent the bending angle of M2$i$, and the increment of the bending angle is given as follows:

$$
\Delta \theta_{i}=\theta_{i}-\theta, \quad(i=1,2, \ldots, 8, \text { in this section }) .
$$

The parameter $R$ is used to represent the curvature radius of the reference actuator, $R_{i}$ represent the curvature radius of $\mathrm{M} 2-i$, and the increment of the curvature radius is given as follows:

$$
\Delta R_{i}=R_{i}-R, \quad(i=1,2, \ldots, 8, \text { in this section }) .
$$

The value of bending angle $\theta$ under different pressures is shown in Figure 5(a) (i.e., the value corresponding to the longitudinal coordinate in the polygonal line $\mathrm{Mr}$ ), and the value of curvature radius $R$ is shown in Figure 5(c) (i.e., the value corresponding to the longitudinal coordinate in the polygonal line $\mathrm{Mr}$ ).

Figure 5(a) displays the bending angle of actuators with different values of $w_{2}$ under different pressures (i.e., 0$35 \mathrm{kPa}$ ). The bending angle of each of the eight actuators increases with the increase of pressure, and it also increases with the increase of $w_{2}$ under the same pressure. Figure 5(b) presents the increment of bending angle between the reference actuator and other actuators. We can see that the increments of each actuator are increased with the increase of pressure. The increments of each actuator are also increased with the increase of $w_{2}$ under the same pressure. It means that 

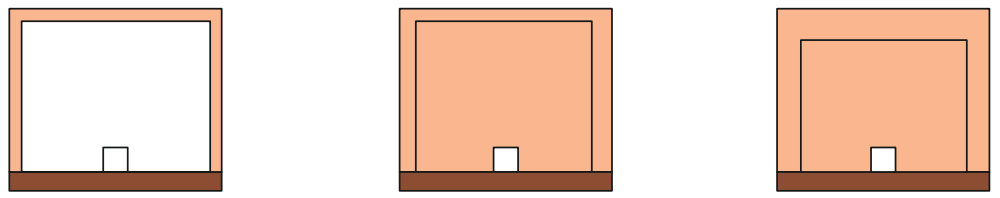

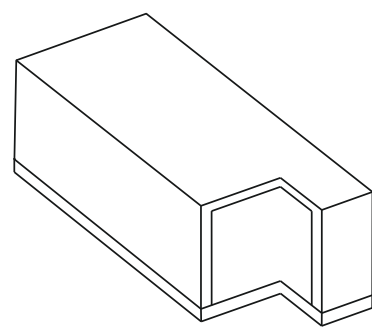

(a)

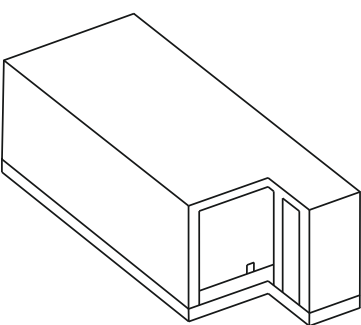

(b)

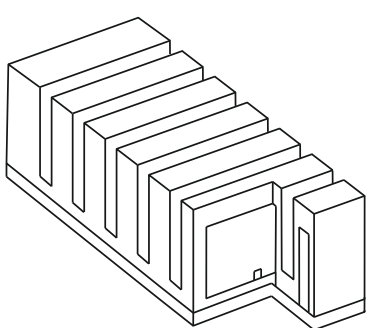

(c)

FIGURE 1: Different structures of soft pneu-net actuators. (a) Actuators with a single chamber. (b) Actuators with a network of chambers without channel between two chambers. (c) Actuators with a network of chambers and uniformly distributed channels.

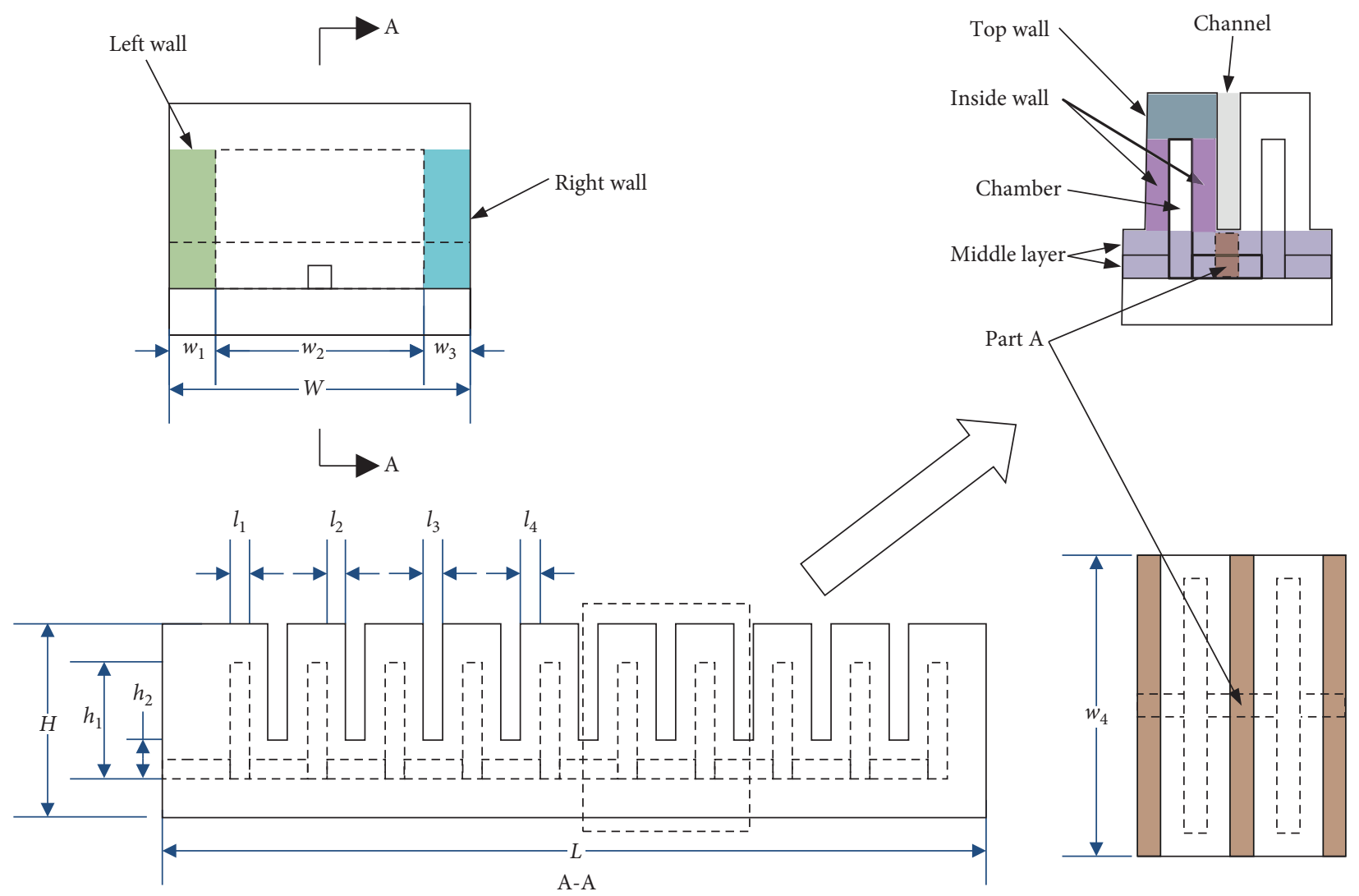

FIGURE 2: Structure of the reference actuator.

if the length of an actuator is a certain value, the actuator can change its bending angle with the same pressure by altering the design parameter $w_{2}$.

As shown in Figure 5(c), the curvature radius of each actuator decreases with the increase of pressure. Under a certain pressure, the curvature radius decreases with the increase of $w_{2}$. The increments of curvature radius with increasing $w_{2}$ are displayed in Figure $5(\mathrm{~d})$. The gradients of increments of curvature radius become slow with the increase of pressure. The absolute values of increments of curvature radius increase with the increase of $w_{2}$ under the same pressure. It can be concluded that an actuator can also achieve a smaller curvature radius under the same pressure by increasing $w_{2}$.

A series of actuators (M4-1 M4-8) with different values of the width parameter $w_{4}\left(w_{4-i}=24 \sim 10 \mathrm{~mm}\right)$ are also designed and analysed to explore the sensitivity of the width parameter $w_{4}$ to the bending performance of the actuator. 
TABLE 1: Parameters of the reference actuator.

\begin{tabular}{lc}
\hline Parameter & Values $(\mathrm{mm})$ \\
\hline Length of the actuator $(L)$ & 85 \\
Width of the actuator $(W)$ & 26 \\
Height of the actuator $(H)$ & 20 \\
Length of the chamber $\left(l_{1}\right)$ & 2 \\
Thickness of the inside wall $\left(l_{2}, l_{4}\right)$ & 2 \\
Length of the channel $\left(l_{3}\right)$ & 2 \\
Height of the chamber $\left(h_{1}\right)$ & 12 \\
Height of the middle layer $\left(h_{2}\right)$ & 4 \\
Thickness of the left wall $\left(w_{1}\right)$ & 4 \\
Width of the chamber $\left(w_{2}\right)$ & 18 \\
Thickness of the right wall $\left(w_{3}\right)$ & 4 \\
Width of the middle layer $\left(w_{4}\right)$ & 26 \\
\hline
\end{tabular}

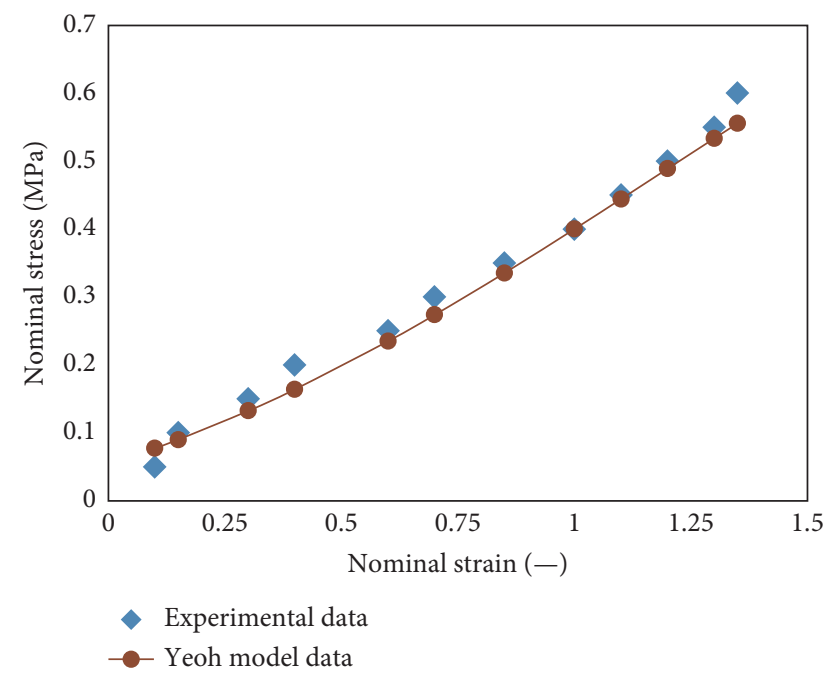

FIGURE 3: Measurement of the stress-strain relation of Dragon Skin 30 silicone rubber obtained from the uniaxial tensile test. The experimental data are fitted with the Yeoh model $(C 10=0.0709$, $C 20=-0.01015$, and $C 30=0.0002)$.

It can be seen that the bending angle of each of the eight actuators increases with the decrease of $w_{4}$ under the same pressure in Figure 6(a). For a given actuator, the bending angle increases with the increase of pressure. Figure 6(b) presents the increment of bending angle between the reference actuator and other actuators. The increments of bending angle of each actuator are also increased with the increase of pressure. As shown in Figure 6(b), when the actuators are subjected to the same pressure, the increments of bending angle increase with the decrease of $w_{4}$. Figure 6(c) displays that the curvature radius increases with the increase of $w_{4}$ under the same pressure. The relation between the increment of curvature radius and the increment of $w_{4}$ is displayed in Figure 6(d). The absolute value of increments of curvature radius increases with the decrease of $w_{4}$ under the same pressure. It can be concluded that an actuator can also change its bending angle and curvature radius by altering the value of $w_{4}$.

3.2. Optimum Design Method of Pneu-Net Actuator. The effects of the chamber width parameter and the middle-layer width parameter on the bending performance of the actuator under different pressures are discussed in detail in the above section, respectively. We can find that the relation between the increment of the chamber width and the increment of the bending angle is approximately linear under a certain pressure (Figure 5(b)), and the relation between the increment of the middle-layer width and the increment of the bending angle is nonlinear (Figure 6(b)).

However, no matter which width parameter changes, the bending angle increases linearly with the increase of pressure (Figures 5(a) and 6(a)). Moreover, when the pressure is $0 \mathrm{kPa}$, the bending angle is $0 \mathrm{rad}$. Therefore, for any width, there is only one parameter to be determined in the mathematical relationship between bending angle and pressure: the slope that changes according to the width parameter.

Combined with the linear fitting result in Figure 5(a), relative to the reference actuator, we can obtain the corresponding slopes under different increments of $w_{2}$ (Figure 7(a)), and the mathematical relation between them can be obtained by using the cubic polynomial fitting algorithm; in this case, the fitting result is given as follows:

$$
\begin{aligned}
k_{\theta}\left(\Delta w_{2}\right)= & -0.000469 *\left(\Delta w_{2}\right)^{3}+0.00312 *\left(\Delta w_{2}\right)^{2} \\
& +0.0171 *\left(\Delta w_{2}\right)+0.1235 \\
= & f_{1}\left(\Delta w_{2}\right)+0.1235 \\
\Delta w_{2}= & w_{2-i}-w_{\mathrm{r} 2},
\end{aligned}
$$

where $w_{\mathrm{r} 2}$ is the value of the chamber width of the reference actuator and $w_{2-i}$ is the value of the chamber width of any actuator.

Similarly, combined with the linear fitting result in Figure 6(a), the corresponding slopes under different increments of $w_{4}$ are shown in Figure 7(b), and the mathematical relation between them can also be obtained by using the cubic polynomial fitting algorithm; the fitting result is obtained in this case as follows:

$$
\begin{aligned}
k_{\theta}\left(\Delta w_{4}\right)= & 0.0000625 *\left(\Delta w_{4}\right)^{3}+0.00163 *\left(\Delta w_{4}\right)^{2} \\
& -0.00335 *\left(\Delta w_{4}\right)+0.1235 \\
= & f_{2}\left(\Delta w_{4}\right)+0.1235 \\
\Delta w_{4}= & w_{4-i}-w_{\mathrm{r} 4},
\end{aligned}
$$

where $w_{\mathrm{r} 4}$ is the value of the middle-layer width of the reference actuator and $w_{4-i}$ is the value of the chamber width of any actuator.

Then, we can get the mathematical relation between the bending angle and the pressure in any width of the chamber or the middle layer:

$$
\begin{aligned}
& \Theta_{P}\left(w_{2-i}\right)=k_{\theta}\left(\Delta w_{2}\right) * P=f_{1}\left(\Delta w_{2}\right) * P+0.1235 * P, \\
& \Theta_{P}\left(w_{4-i}\right)=k_{\theta}\left(\Delta w_{4}\right) * P=f_{2}\left(\Delta w_{4}\right) * P+0.1235 * P,
\end{aligned}
$$

where $P$ represents the pressure.

Combined with equations (5) and (6), because of the change in the width of the chamber and the middle layer relative to the reference actuator, a unified bending model of actuators can be established as follows: 


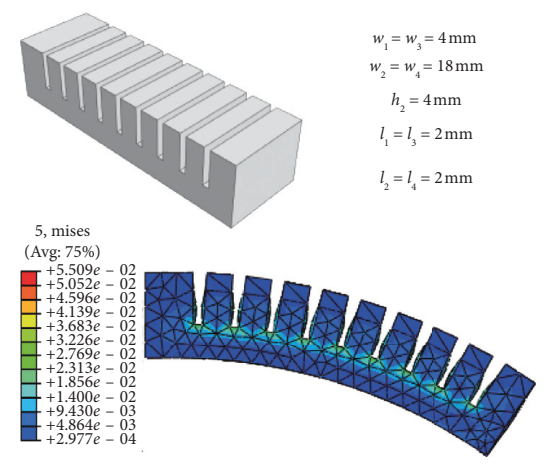

(a)

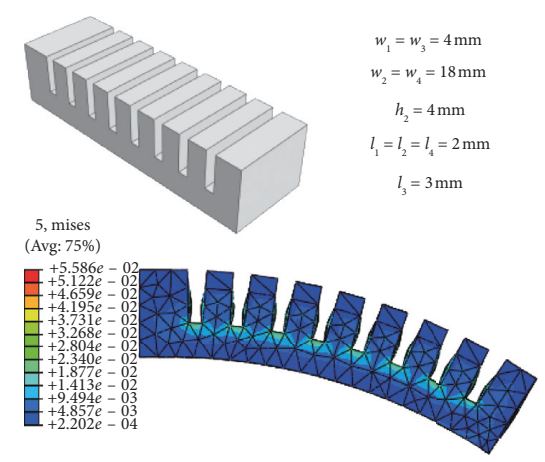

(d)

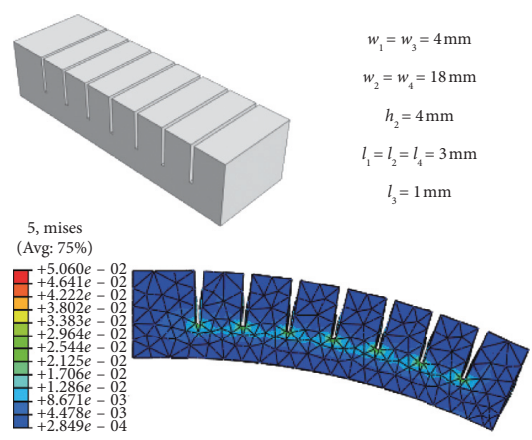

(g)

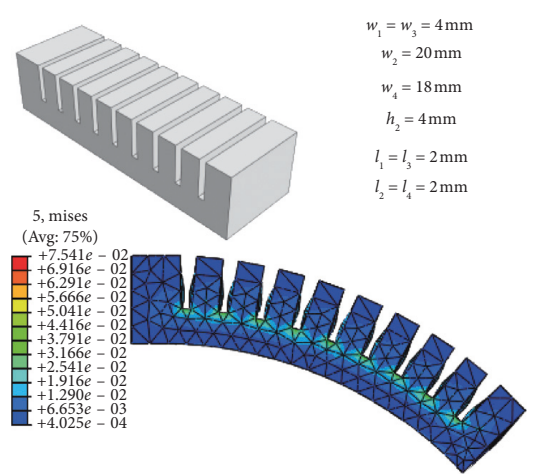

(b)

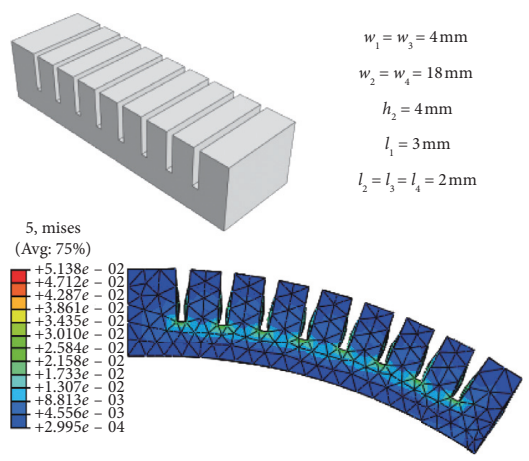

(e)

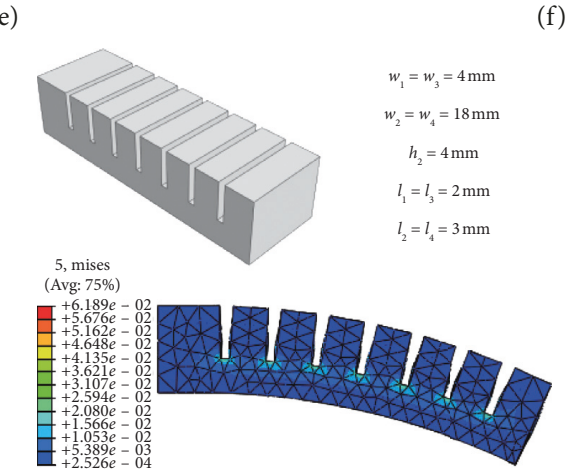

(h)

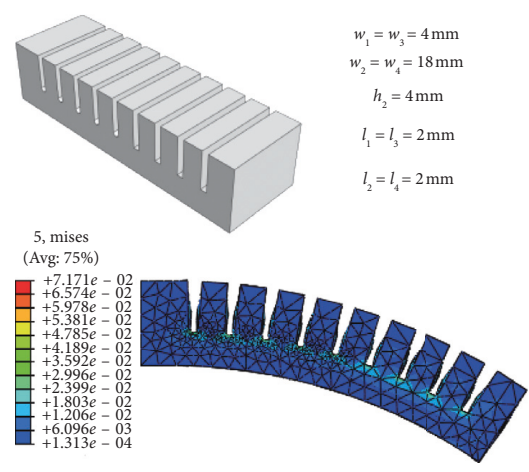

(c)

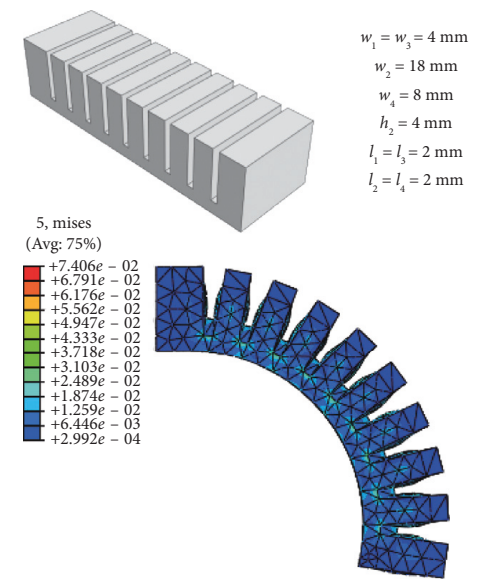

(f)

FIGURE 4: FEA results of the actuators with different parameters under the actuation pressure of $5 \mathrm{kPa}$. The original and deformed shapes of each actuator are illustrated in (a)-(h).

$$
\Theta_{P}=\left(f_{1}\left(\Delta w_{2}\right)+f_{2}\left(\Delta w_{4}\right)+0.1235\right) * P .
$$

The actuator bending model is established according to the results of linear fitting and cubic polynomial fitting, both of which have a higher coefficient of determination $\left(R^{2} \geq 0.99\right)$. The bending model expresses quantitatively the effect of two width parameters on the bending performance of actuators. However, the ultimate goal of adjusting the design parameters is to match the target trajectory, so we will focus on the characteristics of the end trajectory of actuators.

Three actuators with different parameters are selected in Section 2, and the pressure on each actuator is gradually increased so that the bending degree of each of them reaches the limit state (i.e., interference with itself). The results of the three end trajectories are shown in Figure 8. Note that the three actuators that have different design parameters generate different bending angles under the same pressure. Because the three actuators are of the same length, the entire trajectories generated by them are coincident; that is, when length parameters of actuators are the same, the changes of other design parameters will not affect the trend of the end trajectory. Hence, when the operation task needs to match a trajectory of a particular path, a single pneu-net actuator needs to be made up of several interrelated segments, and each segment requires different and appropriate design parameters. 


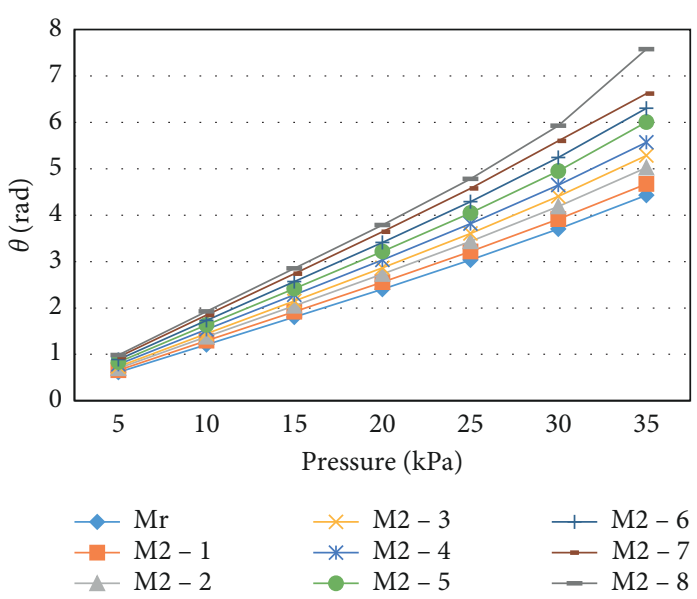

(a)

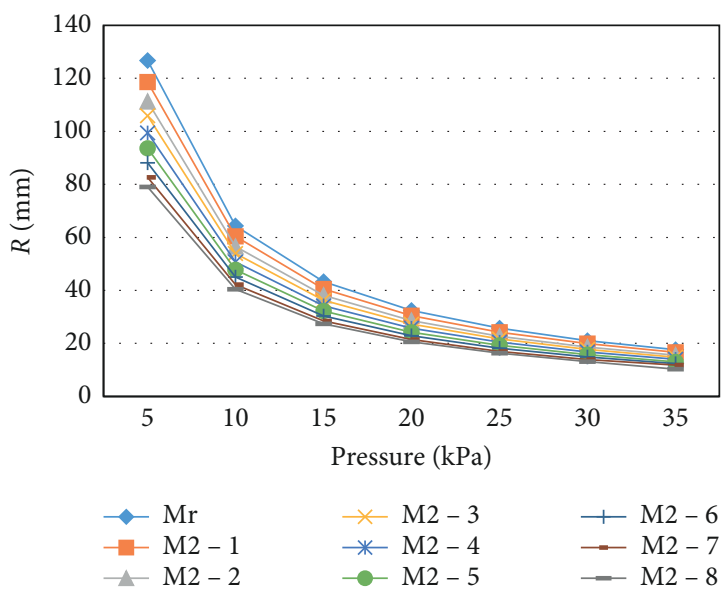

(c)

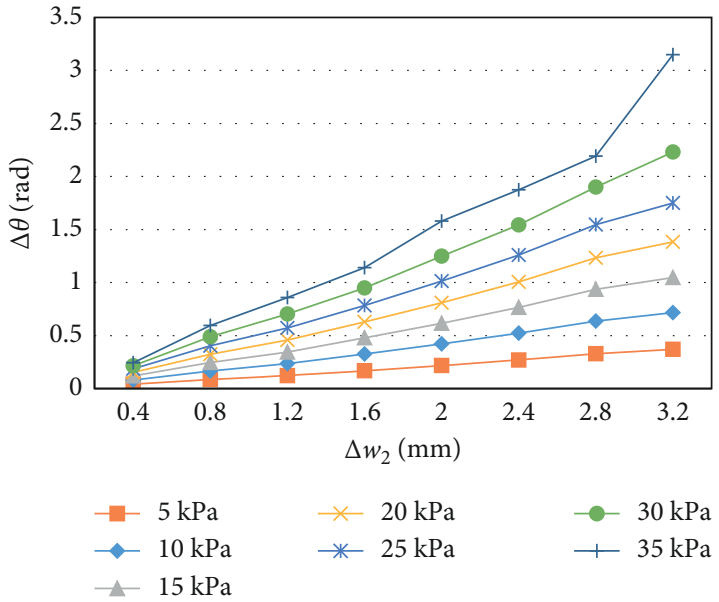

(b)

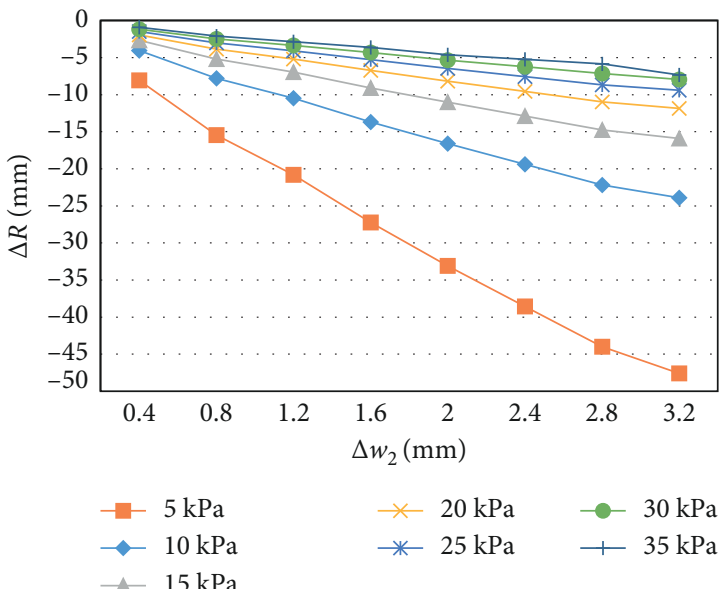

(d)

FIGURE 5: FEA results of the actuators with different $w_{2}$. (a) Bending angle under different pressures. (b) Increments of the bending angle. (c) Curvature radius under different pressures. (d) Increments of the curvature radius.

On the contrary, to achieve target trajectory matching of the pneu-net actuator, three different design parameters need to be taken into account: segment length, chamber width, and middle-layer width. Because the lengths of actuators used in the bending performance experiments are the same as that of the reference actuator (length of the reference actuator $L_{\mathrm{r}}=85 \mathrm{~mm}$ ), in order to quantitatively analyse the influence of these three crucial design parameters on the bending performance, an additional comparative experiment is designed. The actuators in this experiment have only the variable-length parameter, and the other parameters are consistent with those of the reference actuator.

It can be seen from Figure 9 that the relation between the radius of the curvature and the pressure of the actuators with different length parameters is approximately the same. Hence, for actuators with different length parameters, we can use the constant characteristic of the radius of the curvature to represent the bending angle. According to equation (7), the radius of the curvature of the actuator can be expressed as

$$
R_{\mathrm{r}}(P)=\frac{L_{\mathrm{r}}}{\Theta_{P}}=\frac{L_{\mathrm{r}}}{\left(f_{1}\left(\Delta w_{2}\right)+f_{2}\left(\Delta w_{4}\right)+0.1235\right) * P} .
$$

When the length parameter $L$ is changed, according to the constant characteristic of the radius of the curvature, the bending model of the actuator can be expressed as

$$
\Theta_{P}^{*}=\frac{L}{L_{\mathrm{r}}} * \Theta_{P}
$$

For a given target trajectory, the length parameter of the actuator is a determined parameter, i.e., a constraint condition. However, the number of segments, the length of each segment, and the corresponding two width parameters of the desired actuator need to be optimized. To tackle that problem, a multiobjective optimization algorithm based on the genetic algorithm (GA) is proposed as an automatic search optimization technology, which automatically adjusts the design parameters to achieve the optimization goal.

The search of the GA for the desired actuator with better predictive capabilities is treated as a minimization problem with the mean square error (MSE) as an adaptation value, 


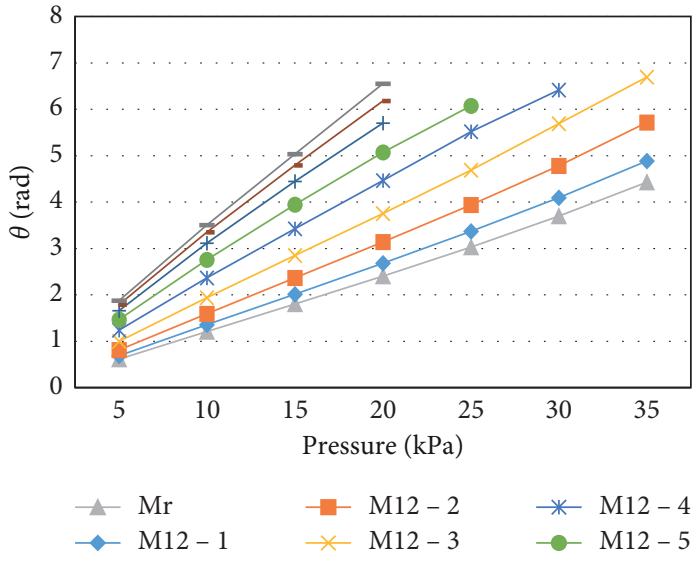

(a)

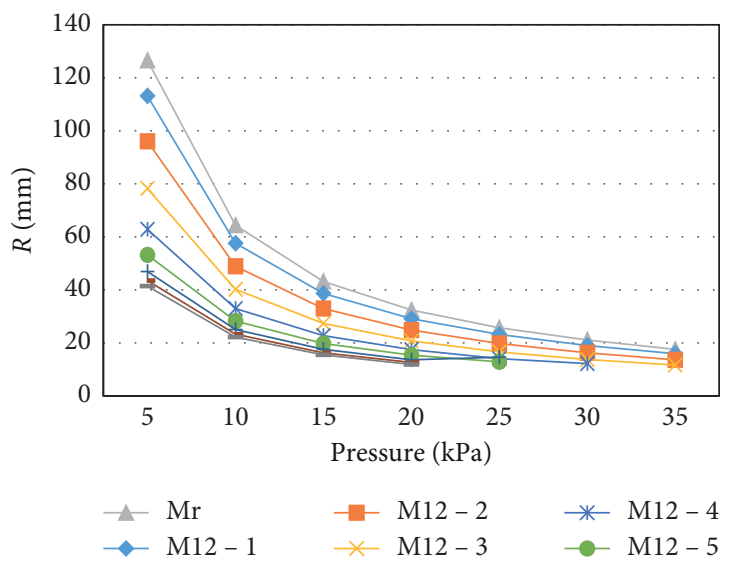

(c)

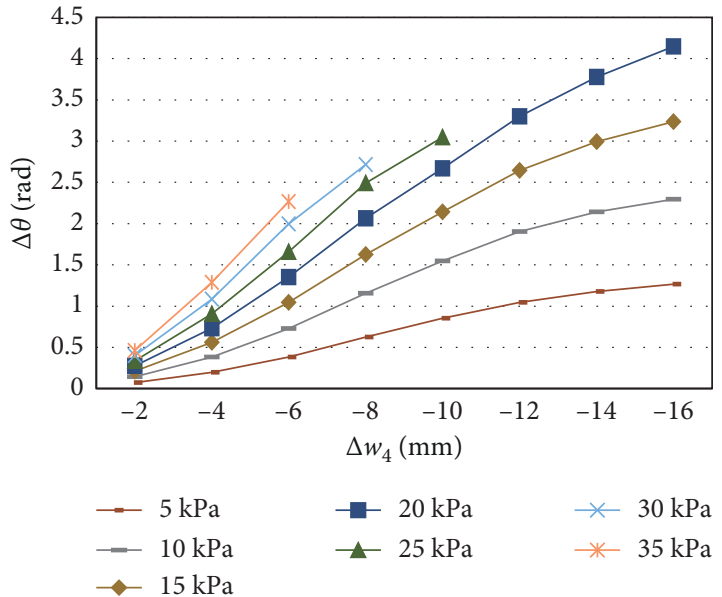

(b)

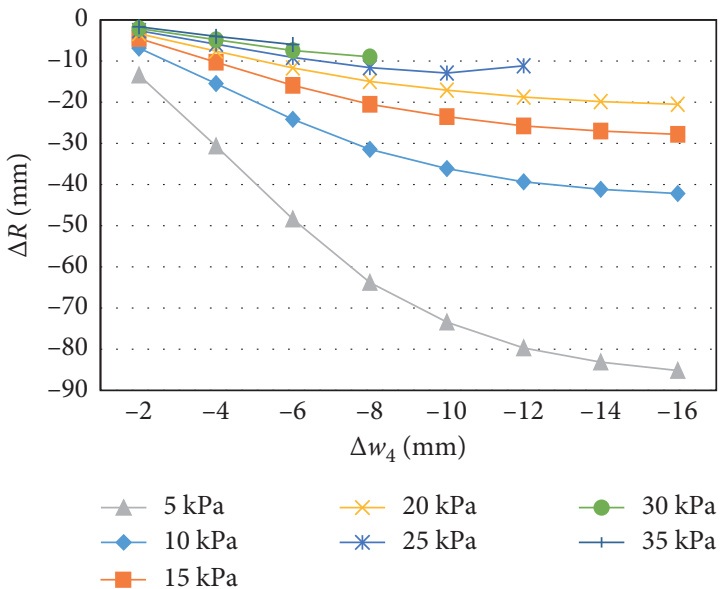

(d)

Figure 6: FEA results of the actuators with different $w_{4}$. (a) Bending angle under different pressures. (b) Increments of the bending angle. (c) Curvature radius under different pressures. (d) Increments of the curvature radius.

which refers to the error between the design trajectory and the target trajectory. Taking the head of the desired pneu-net actuator as the origin of the base coordinate system, the design trajectory can be calculated based on the homogeneous coordinate transformation method:

$$
\left[\begin{array}{lll}
x & y & 1
\end{array}\right]=\left[\begin{array}{lll}
0 & 0 & 1
\end{array}\right]\left[\begin{array}{ccc}
1 & 0 & 0 \\
0 & 1 & 0 \\
x_{1} & y_{1} & 1
\end{array}\right]\left[\begin{array}{ccc}
1 & 0 & 0 \\
0 & 1 & 0 \\
x_{2} & y_{2} & 1
\end{array}\right] \cdots\left[\begin{array}{ccc}
1 & 0 & 0 \\
0 & 1 & 0 \\
x_{n} & y_{n} & 1
\end{array}\right] \text {, }
$$

where

$$
\left\{\begin{array}{l}
x_{i}=R_{i}(P) \cdot \sin \left(\Theta_{P}^{*}\right) \\
y_{i}=R_{i}(P) \cdot\left(1-\cos \left(\Theta_{P}^{*}\right)\right) \\
i=1,2, \ldots, n, \quad n \text { is the number of segments. }
\end{array}\right.
$$

The parameters of the GA are set as follows: the size of the population is 80 ; the crossover rate and mutation rate are 0.8 and 0.05 , respectively; the number of generations is 300 ; and the fitness function of the GA for the design trajectory is presented as

$$
\min f=\frac{1}{m} \sum_{j=1}^{k}\left(\left(x_{j}-\hat{x}_{j}\right)^{2}+\left(y_{j}-\hat{y}_{j}\right)^{2}\right),
$$

where $m$ is the number of comparison points selected in the target trajectory.

Hence, the optimum design method of the pneu-net actuator proposed in this work consists of two steps: (1) With the results of FEA, more sensitive design parameters are determined and the bending model of the actuator is established. (2) According to the design objectives, the GA is used to optimize these three crucial design parameters (i.e., segment length, chamber width, and middle-layer width) to provide a set of optimal geometric parameters for the optimum design of the pneu-net actuator. In the next section, an example will be used to illustrate the feasibility and effectiveness of our proposed method. 


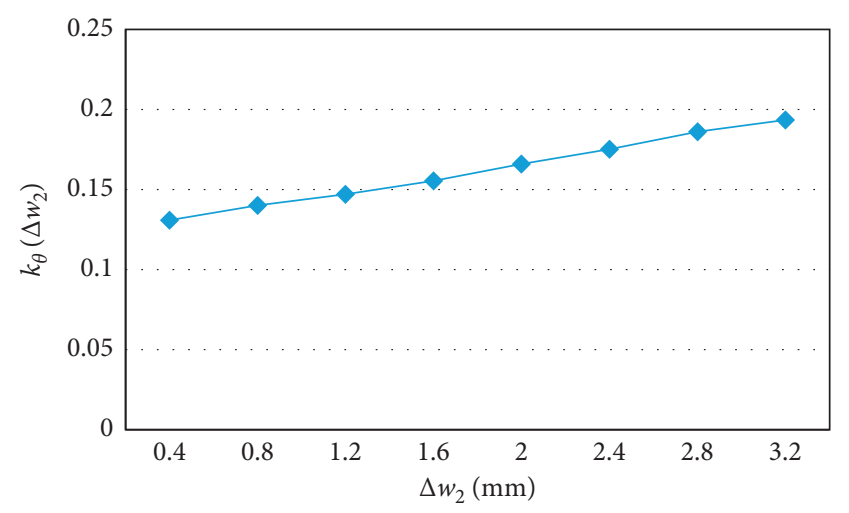

(a)

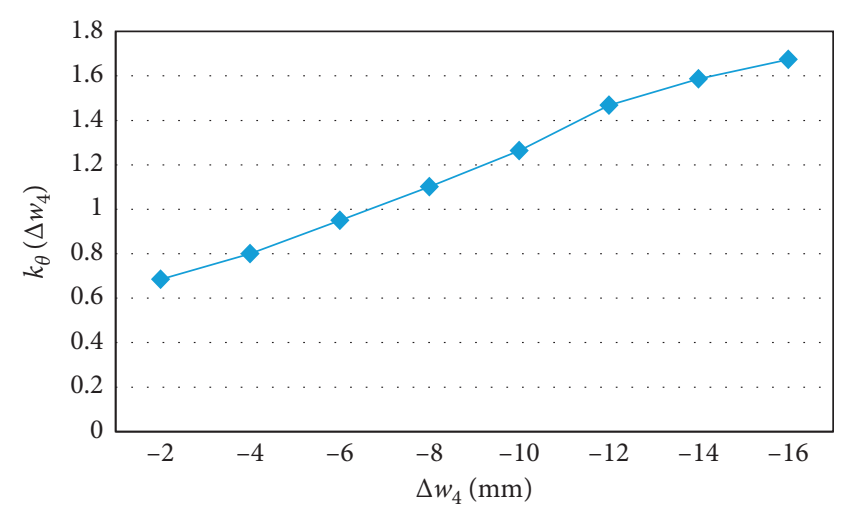

(b)

FIgURE 7: Slopes under different width increments. (a) Increments of $w_{2}$. (b) Increments of $w_{4}$.

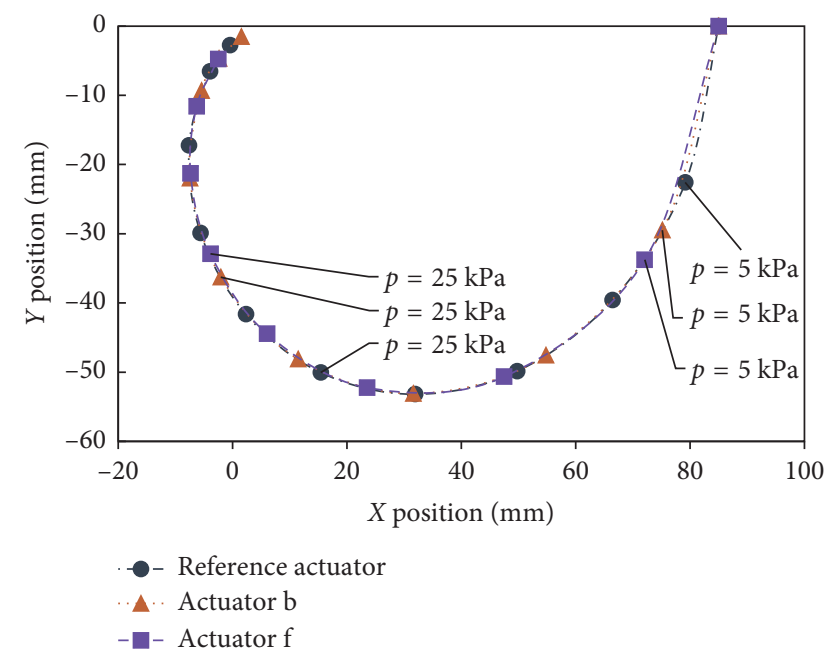

FIGURE 8: The end trajectory of actuators designed with different design parameters.

\section{Experimental Results and Discussion}

To verify the effects of the optimum design method on the performance of the matching-specific target trajectory, an actuator is designed by the grasping motion of a human index finger. The index finger contains metacarpophalangeal, interphalangeal, and proximal interphalangeal joints, in which each joint generates different bending angles when completing a grasping motion. Moreover, the index finger consists of three joints connected by three links, respectively, and each segment of the actuator can be regarded as a combination of one joint and one link. To obtain the specific trajectory of the grasping motion of an index finger, one index finger is photographed to record the configurations (the length of each link and the angles between the links) and the trajectory (Figures 10(a), 10(b), and $10(d))$.

On the basis of the obtained target trajectory, we need to ensure that each segment of the desired actuator can reach their respective target bending angle under the same

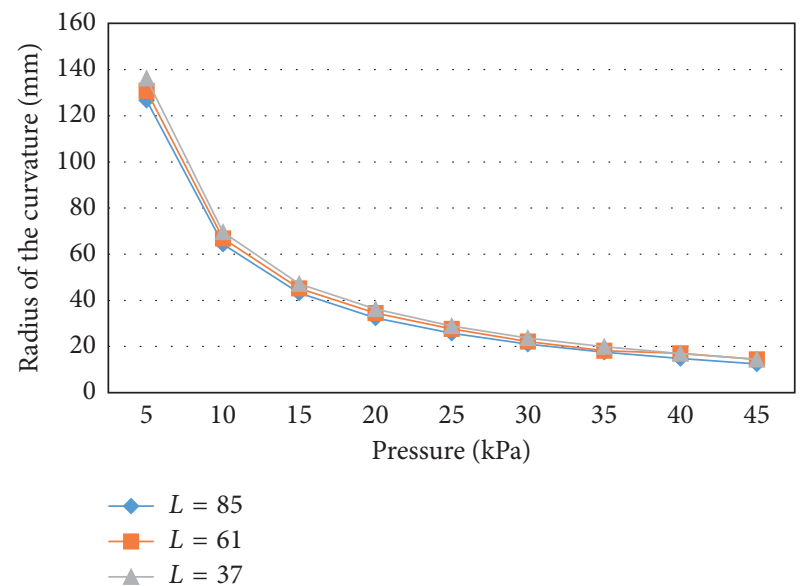

Figure 9: Curvature radius of actuators designed with different actuator lengths.

pressure, and then the desired actuator can accurately match the target trajectory. To match the target trajectory, the GA-based optimum design method is used to design the desired actuator by adjusting the values of segment length, chamber width, and middle-layer width. The parameters of the designed actuator are illustrated in Figure 10(c): the lengths of segments 1,2 , and 3 are 24,26 , and $45 \mathrm{~mm}$, respectively; segment 1 has a chamber width of $3 \mathrm{~mm}$, segment 2 has a middle-layer width of $18 \mathrm{~mm}$, and other parameters are the same as those of the reference actuator. Using finite element simulation, the designed actuator which consists of these three segments with these different segment lengths and design parameters can achieve different bending performances to match the configuration of the index finger.

A casting method is adopted to manufacture the designed actuator. The moulds for the designed actuator are designed in SolidWorks, and these moulds are made by 3D printing technology. First, Smooth-On Dragon Skin 30 silicone parts A and B are regularly mixed and placed in a vacuum box (Figure 11). After all the bubbles are dispelled, the silicone mixture is poured onto the moulds. As shown in 


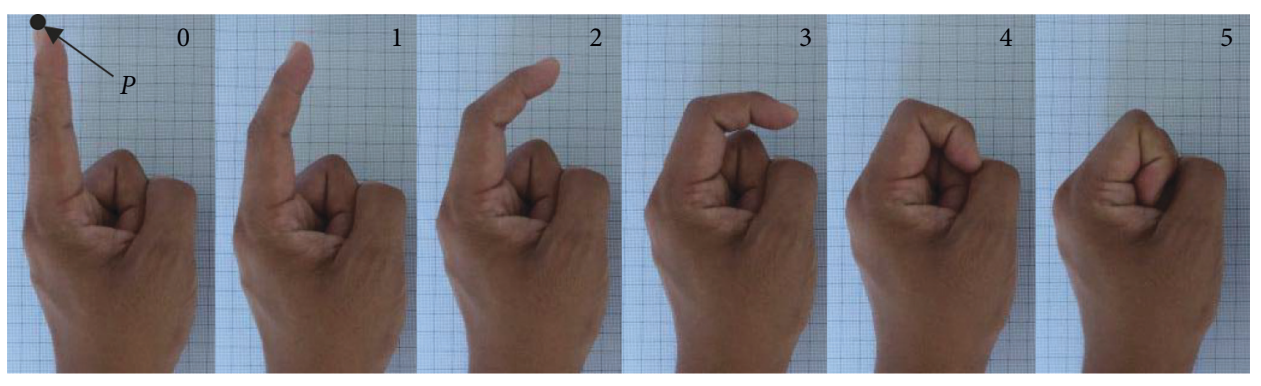

(a)

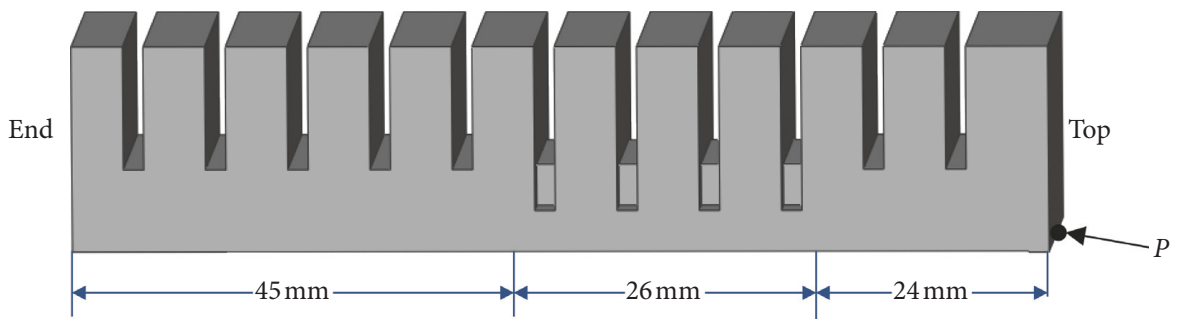

(c)

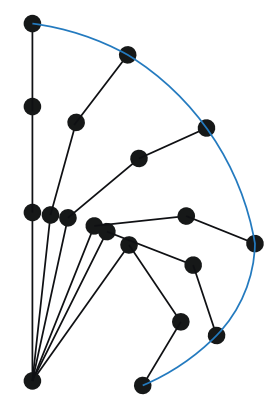

(b)

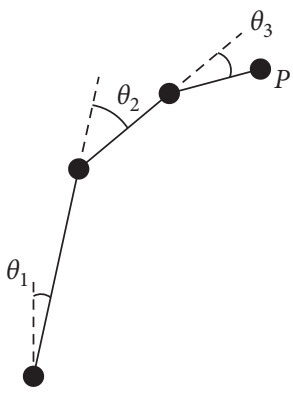

(d)

Figure 10: Generation of the target trajectory and structure of the actuator. (a) Photographs of the index finger during a grasping motion. (b) Configurations of the index finger during a grasping motion. (c) Structure and design parameters of the desired actuator. (d) Illustration of the bending angle of each joint.

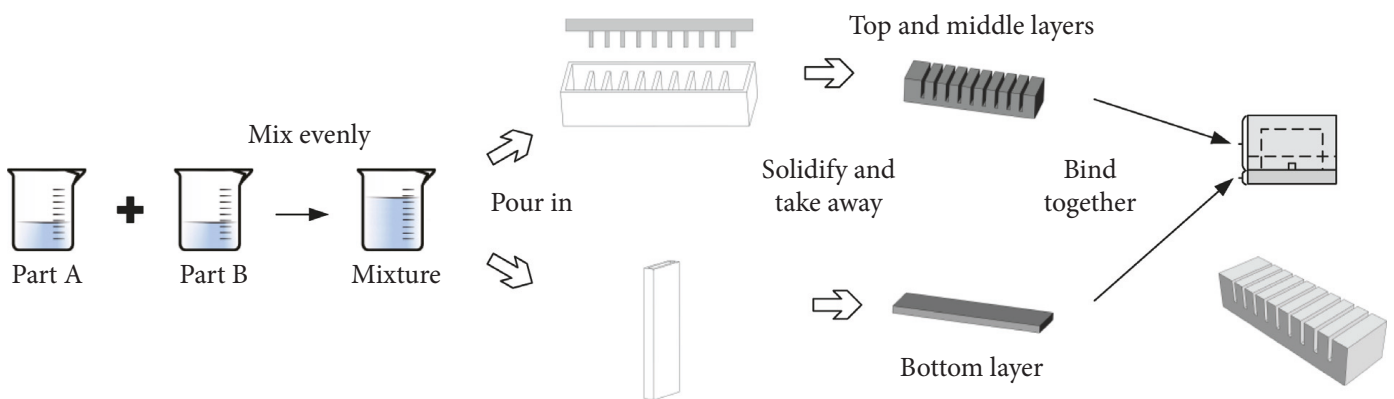

FIGURE 11: Fabrication of the actuator.

Figure 11, the designed actuator is divided into two parts that are separately manufactured by using corresponding moulds and are bonded together when the silicone mixture was solidified.

To verify the actual performance, we capture pictures of the designed actuator under different pressures to calculate the bending angle and length of each segment (Figures 12(a) and $12(\mathrm{~d}))$. The results displayed in Figure 12(b) demonstrate that the actual bending angle of the actuator and target bending angles of the index finger are accurately matched during a grasping motion. Comparing the target trajectory with the actual trajectory of the actuator in Figure 12(c), we can see that most of the two trajectories have a good coincidence degree, but a small part of them does not coincide completely in the initial stage of the path, which is the result of the performance of the actuator itself. Each segment of the actuator has uniformly distributed chambers to drive the actuator achieve bending motion, which causes the link to get shortened under pressure. Thus, each segment must be designed to be slightly longer than the desired segment, thereby indicating that the actual trajectory may not exactly match each point on the target trajectory, especially without pressure. Moreover, as shown in Figure 12(c), a singlesegment actuator cannot achieve this target trajectory matching.

Furthermore, a series of grasping experiments are performed to verify that the designed actuator can simulate grasping motions of the index finger. From the experimental results displayed in Figure 13, it can be seen that the actuator can grasp different relatively small objects, such as a bottle, mould, and ping-pong ball. Hence, these experiments prove that the actuator can achieve target trajectory matching and complete the stable grasping of different objects. 

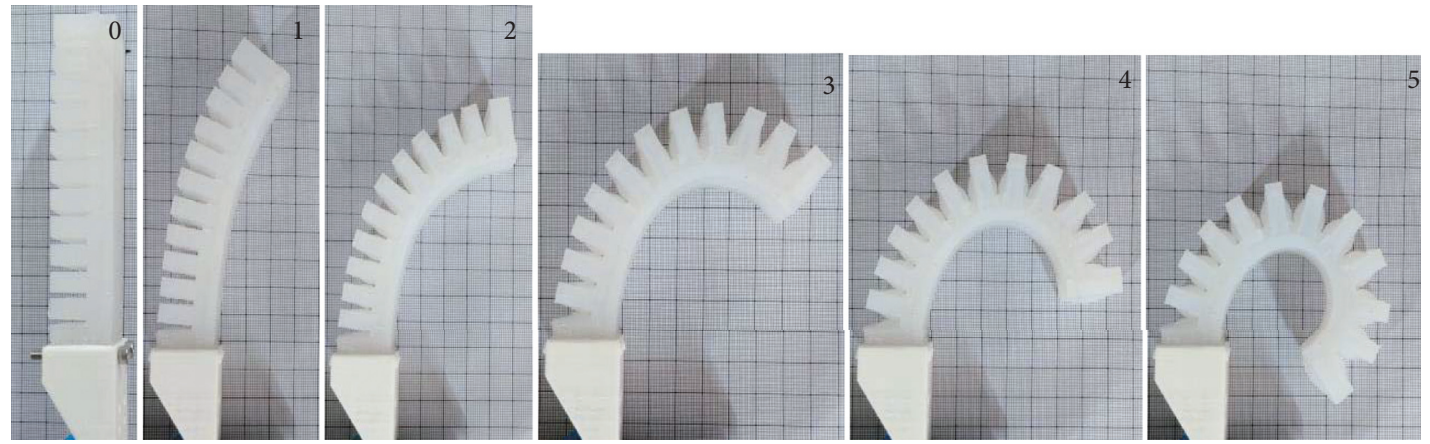

(a)

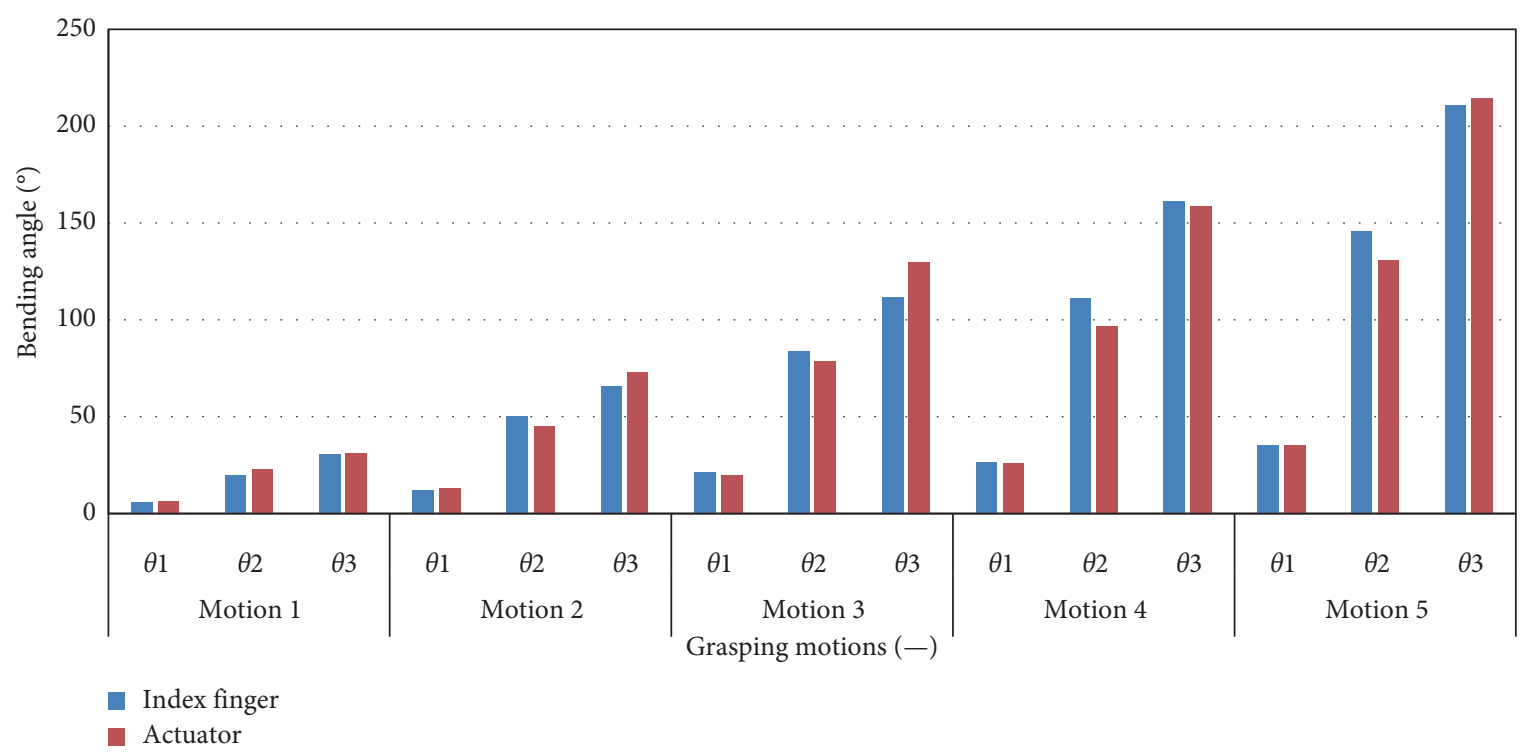

(b)

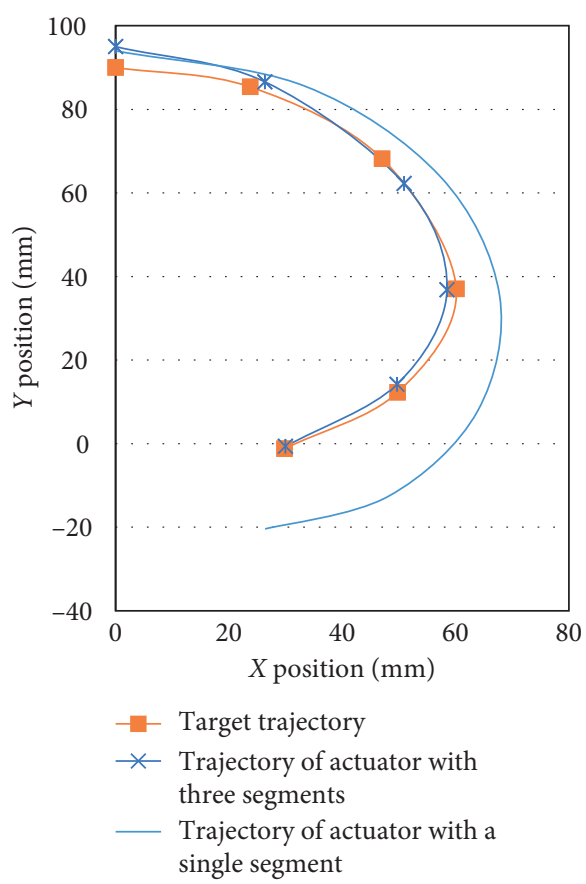

(c)

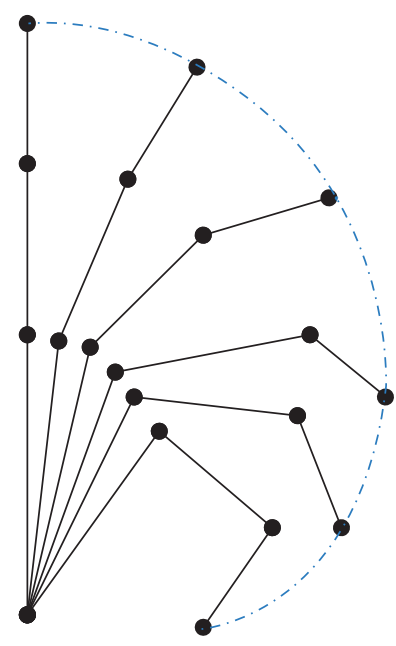

(d)

Figure 12: Experimental results of the designed actuator. (a) Photographs of the actuator during a bending motion. (b) Bending angles of each joint of the index finger and the actuator during a grasping motion. (c) Comparison of trajectories. (d) Configurations of the actuator during a bending motion. 


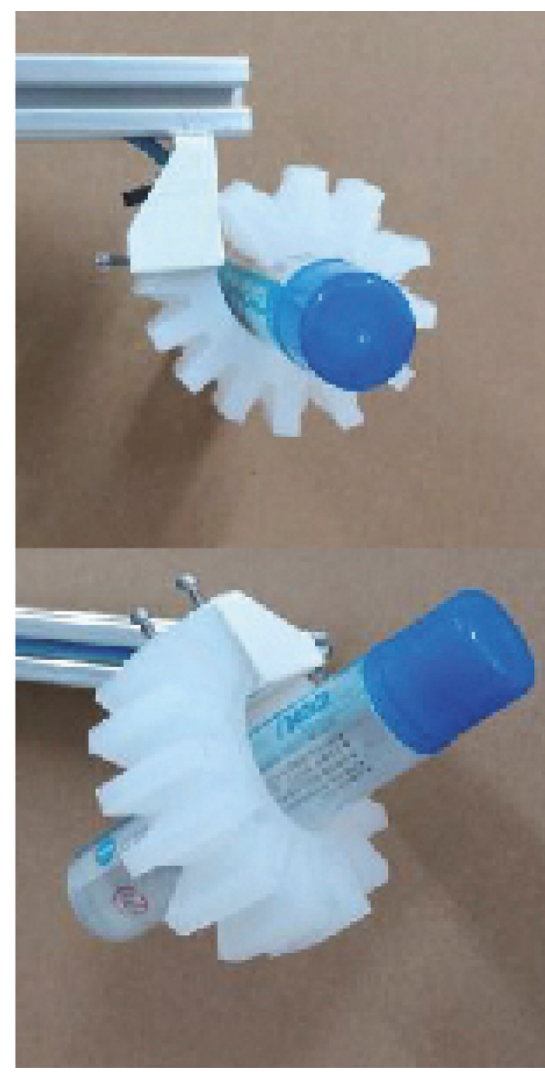

(a)

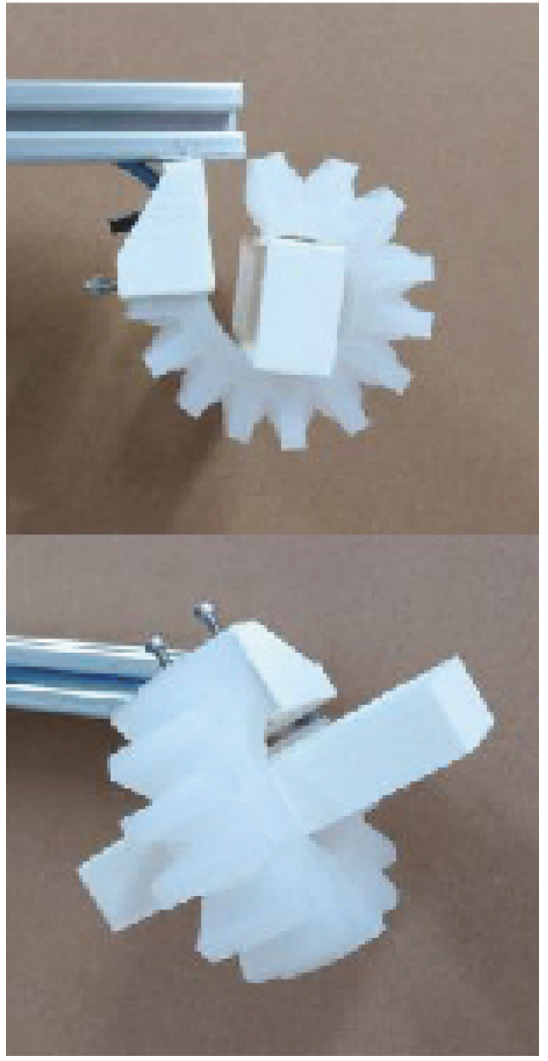

(b)

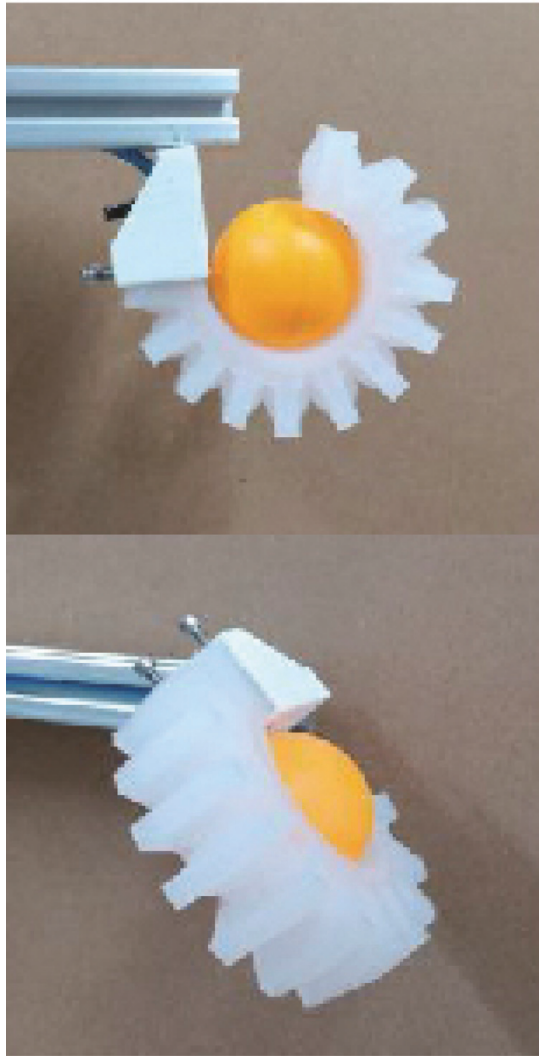

(c)

Figure 13: Grasp experiments for the designed actuator. (a) Bottle with a diameter of $25 \mathrm{~mm}$ and length of $127 \mathrm{~mm}$. (b) Mould with a length of $99 \mathrm{~mm}$, width of $30 \mathrm{~mm}$, and height of $22 \mathrm{~mm}$. (c) Ping-pong ball with a diameter of $37 \mathrm{~mm}$.

\section{Conclusion}

This study has proposed a method for designing a pneunet actuator that can match the target trajectory by adjusting the design parameters. Based on the technology of FEA, we can see that the effects of the chamber width parameter and the middle-layer width parameter on the bending performance of actuators are more sensitive than those of other parameters. Besides, no matter which width parameter changes, the bending angle increases linearly with the increase of pressure. According to the relation between the bending angle and the increment of width, a bending model of the pneu-net actuator is established with the results of linear fitting and cubic polynomial fitting, and both fitting results have higher $R^{2}$ (i.e., $\left.R^{2} \geq 0.99\right)$.

Because the trajectories generated under the actuators with different design parameters are the same when the lengths of actuators are the same, we propose a segmental optimal design method for the desired actuator. To match the target trajectory, the number of segments, the length of each segment, and the corresponding two width parameters of the chamber and middle layer are optimized by using the GA. The experimental results demonstrate that the actuator which is designed on the basis of the abovementioned method can accurately match the target trajectory generated in accordance with the specific trajectory of an index finger during a grasping motion. Furthermore, the designed actuator can perform stable grasping by simulating the grasping motions of the index finger.

Thus, the proposed optimum design of the pneu-net actuator method can improve the performance and give a solution for designing pneu-net actuators for the target task, thereby extending their applications in the field of soft grippers, crawling robots, and bionic robots. The performance of the pneu-net actuator is slightly limited because it can only generate bending motions. Thus, our future work aims to combine several segments that can collectively generate bending, twisting, and extending motions in a single actuator to achieve various trajectories.

\section{Data Availability}

No data were used to support this study.

\section{Conflicts of Interest}

The authors declare that there are no conflicts of interest regarding the publication of this paper.

\section{Acknowledgments}

This research was supported by the National Natural Science Foundation of China (No. 51575236) and Postgraduate 
Research \& Practice Innovation Program of Jiangsu Province (No. KYCX18_1840).

\section{References}

[1] R. Mutlu, G. Alici, and W. Li, "An effective methodology to solve inverse kinematics of electroactive polymer actuators modelled as active and soft robotic structures," Mechanism and Machine Theory, vol. 67, pp. 94-110, 2013.

[2] J. Shintake, S. Rosset, B. Schubert, D. Floreano, and H. Shea, "Versatile soft grippers with intrinsic electroadhesion based on multifunctional polymer actuators," Advanced Materials, vol. 28, no. 2, pp. 231-238, 2016.

[3] R. Yoshida, "Self-oscillating gels driven by the BelousovZhabotinsky reaction as novel smart materials," Advanced Materials, vol. 22, no. 31, pp. 3463-3483, 2010.

[4] L. Migliorini, T. Santaniello, Y. Yan, C. Lenardi, and P. Milani, "Low-voltage electrically driven homeostatic hydrogel-based actuators for underwater soft robotics," Sensors and Actuators B: Chemical, vol. 228, no. 2, pp. 758-766, 2016.

[5] R. Yoshida and Y. Murase, "Self-oscillating surface of gel for autonomous mass transport," Colloids and Surfaces B: Biointerfaces, vol. 99, pp. 60-66, 2012.

[6] R. V. Martinez, A. C. Glavan, C. Keplinger, A. I. Oyetibo, and G. M. Whitesides, "Soft actuators and robots that are resistant to mechanical damage," Advanced Functional Materials, vol. 24, no. 20, pp. 3003-3010, 2014.

[7] F. Connolly, C. J. Walsh, and K. Bertoldi, "Automatic design of fiber-reinforced soft actuators for trajectory matching fiberreinforced soft actuators for trajectory matching," Proceedings of the National Academy of Sciences, vol. 114, no. 1, pp. 51-56, 2017.

[8] T. Wang, L. Ge, and G. Gu, "Programmable design of soft pneu-net actuators with oblique chambers can generate coupled bending and twisting motions," Sensors and Actuators A: Physical, vol. 271, no. 1, pp. 131-138, 2018.

[9] D. Rus and M. T. Tolley, "Design, fabrication and control of soft robots," Nature, vol. 521, no. 7553, pp. 467-475, 2015.

[10] S. Kim, C. Laschi, and B. Trimmer, "Soft robotics: a bioinspired evolution in robotics," Trends in Biotechnology, vol. 31, no. 5, pp. 287-294, 2013.

[11] X. You, S. Liu, and C. Zhang, "An improved ant colony system algorithm for robot path planning and performance analysis," International Journal of Robotics and Automation, vol. 33, no. 5, pp. 527-533, 2018.

[12] Y. Dong, H. Xia, Y. Zhu, Q.-Q. Ni, and Y. Fu, "Effect of epoxygraft-polyoxyethylene octyl phenyl ether on preparation, mechanical properties and triple-shape memory effect of carbon nanotube/water-borne epoxy nanocomposites," Composites Science and Technology, vol. 120, pp. 17-25, 2015.

[13] J. Hasan, R. J. Crawford, and E. P. Ivanova, "Antibacterial surfaces: the quest for a new generation of biomaterials," Trends in Biotechnology, vol. 31, no. 5, pp. 295-304, 2013.

[14] J. Lee, D. Bhattacharyya, M. Q. Zhang, and Y. C. Yuan, "Mechanical properties of a self-healing fibre reinforced epoxy composites," Composites Part B: Engineering, vol. 78, pp. 515-519, 2015.

[15] A. Zolfagharian, A. Z. Kouzani, S. Y. Khoo, A. A. A. Moghadam, I. Gibson, and A. Kaynak, "Evolution of 3D printed soft actuators," Sensors and Actuators A: Physical, vol. 250, pp. 258-272, 2016.

[16] P. Panagiotis, Z. Wang, K. C. Galloway, R. J. Wood, and C. J. Walsh, "Soft robotic glove for combined assistance and at-home rehabilitation," Robotics and Autonomous Systems, vol. 73, pp. 135-143, 2015.

[17] B. Mosadegh, P. Polygerinos, C. Keplinger et al., "Pneumatic networks for soft robotics that actuate rapidly," Advanced Functional Materials, vol. 24, no. 15, pp. 2163-2170, 2014.

[18] B. Wang, A. McDaid, M. Biglari-Abhari, T. Giffney, and K. Aw, "A bimorph pneumatic bending actuator by control of fiber braiding angle," Sensors and Actuators A: Physical, vol. 257, no. 15, pp. 173-184, 2017.

[19] R. Deimel and O. Brock, "A novel type of compliant and underactuated robotic hand for dexterous grasping," International Journal of Robotics Research, vol. 35, no. 1-3, pp. 161-185, 2016.

[20] R. V. Martinez, J. L. Branch, C. R. Fish et al., "Robotic tentacles with three-dimensional mobility based on flexible elastomers," Advanced Materials, vol. 25, no. 2, pp. 205-212, 2013.

[21] M. T. Tolley, R. F. Shepherd, B. Mosadegh et al., "A resilient, untethered soft robot," Soft Robotics, vol. 1, no. 3, pp. 213-223, 2014.

[22] L. Wang and C. Luo, "A hybrid genetic tabu search algorithm for mobile robot to solve AS/RS path planing," International Journal of Robotics and Automation, vol. 33, no. 2, pp. 161168, 2018.

[23] Z. Yu, Y. Shi, J. Xie, S. X. Yang, and Z. Dai, "Design and analysis of a bionic adhesive foot for gecko robot climbing the ceiling," International Journal of Robotics and Automation, vol. 33, no. 4, pp. 445-454, 2017.

[24] M. Wehner, R. L. Truby, D. J. Fitzgerald et al., "An integrated design and fabrication strategy for entirely soft, autonomous robots," Nature, vol. 536, no. 7617, pp. 451-455, 2016.

[25] D. Yao, Y. Wu, Y. Wang, and X. Xiao, "Experimental validation of a control method for underactuated bipedal walking on compliant group," International Journal of Robotics and Automation, vol. 33, no. 5, pp. 552-558, 2018.

[26] I. Hussain, G. Salvietti, G. Spagnoletti et al., "A soft supernumerary robotic finger and mobile arm support for grasping compensation and hemiparetic upper limb rehabilitation," Robotics and Autonomous Systems, vol. 93, pp. 1-12, 2017.

[27] S. Liu, J. Liu, J. Xu, X. Ding, T. Lu, and K. Chen, "Preoperative surgical planning for robot-assisted liver tumour ablation therapy based on collision-free reachable workspaces," International Journal of Robotics and Automation, vol. 33, no. 5, pp. 440-457, 2017.

[28] A. Frutiger, J. T. Muth, D. M. Vogt et al., "Capacitive soft strain sensors via multicore-shell fiber printing," Advanced Materials, vol. 27, no. 15, pp. 2440-2446, 2015.

[29] K. M. de Payrebrune and O. M. O'Reilly, "On the development of rod-based models for pneumatically actuated soft robot arms: a five-parameter constitutive relation," International Journal of Solids and Structures, vol. 120, no. 1, pp. 226-235, 2017. 


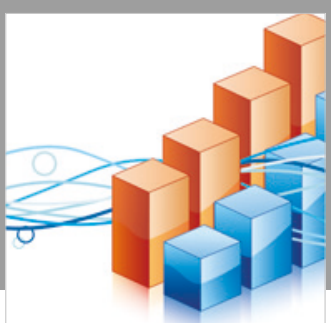

Advances in

Operations Research

\section{-n-m}
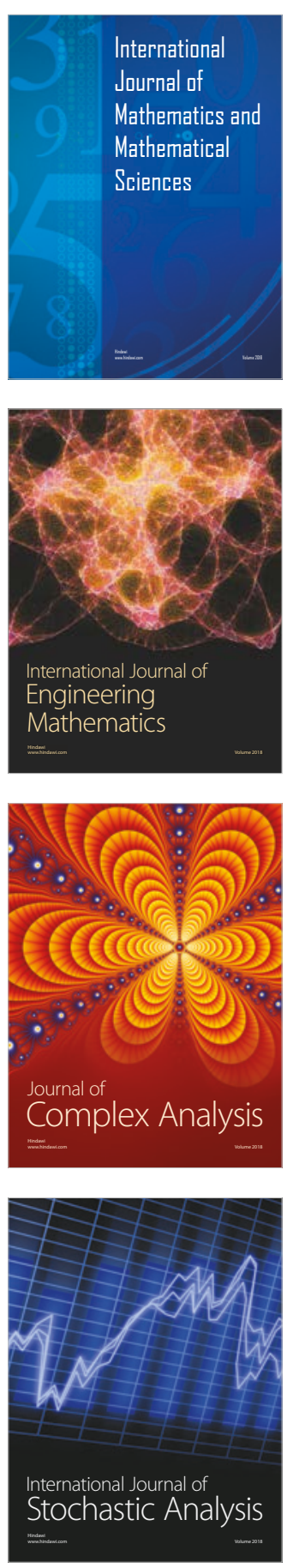
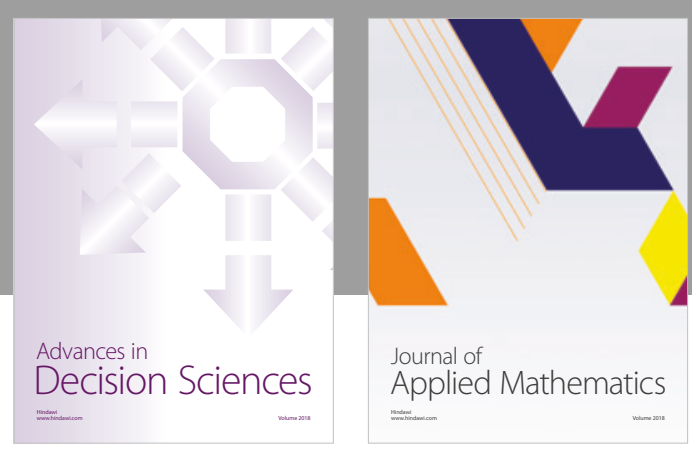

Journal of

Applied Mathematics
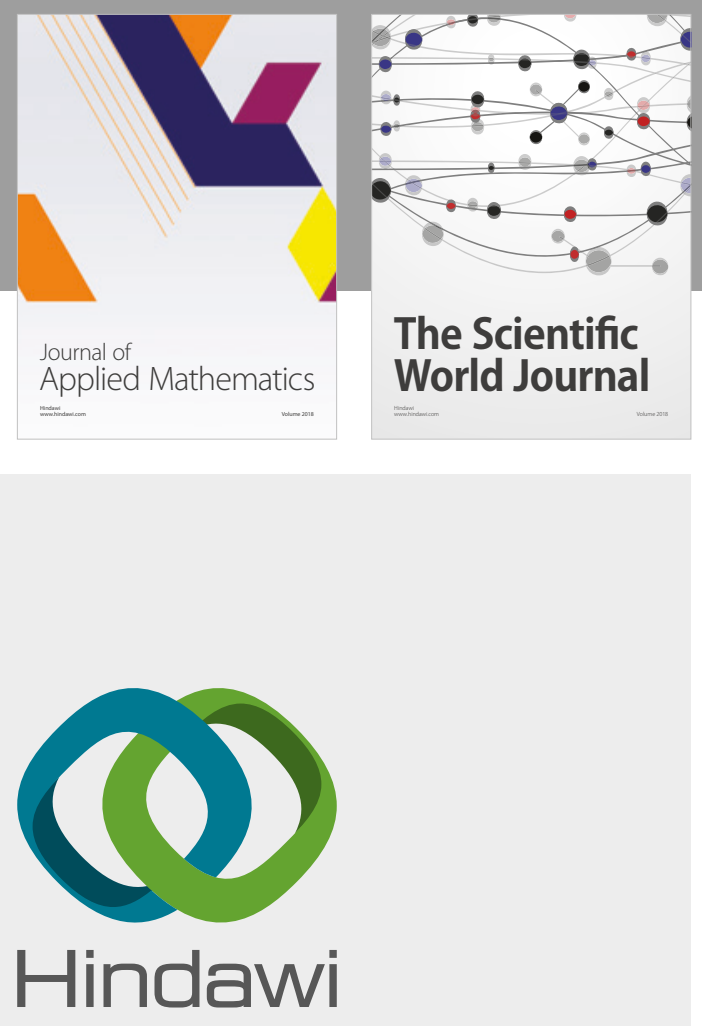

Submit your manuscripts at

www.hindawi.com

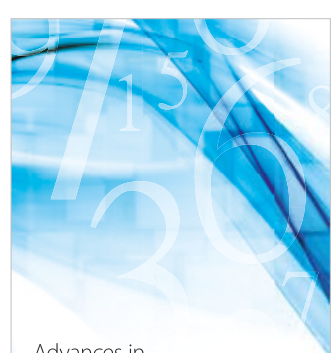

Advances in
Numerical Analysis
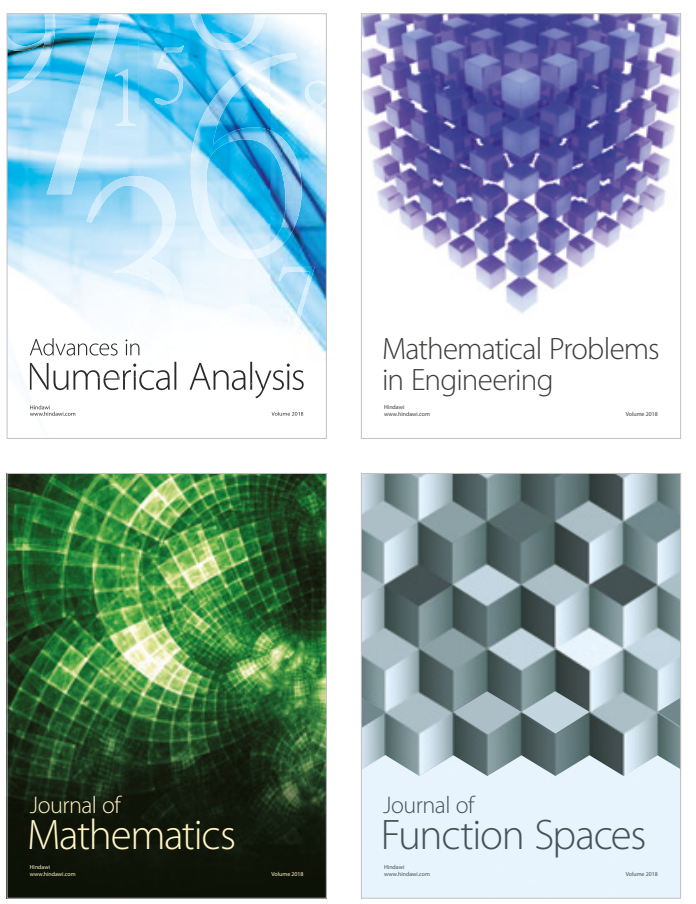

Mathematical Problems in Engineering

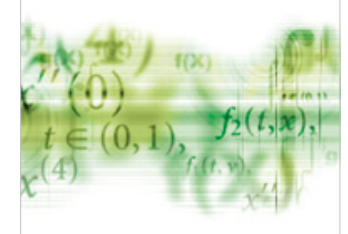

International Journal of

Differential Equations

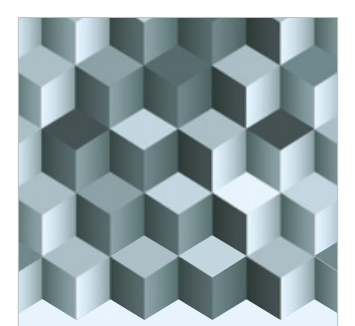

Journal of

Function Spaces

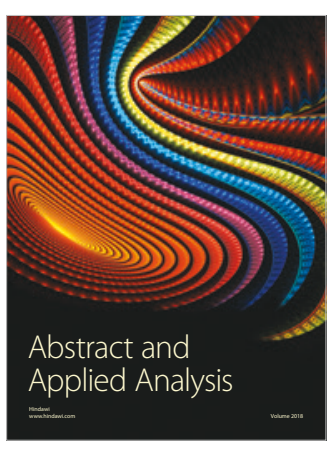

The Scientific

World Journal

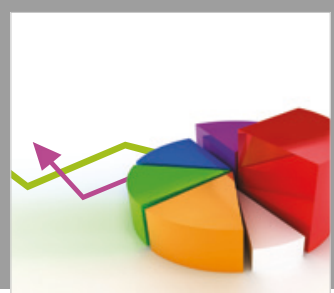

Journal of

Probability and Statistics
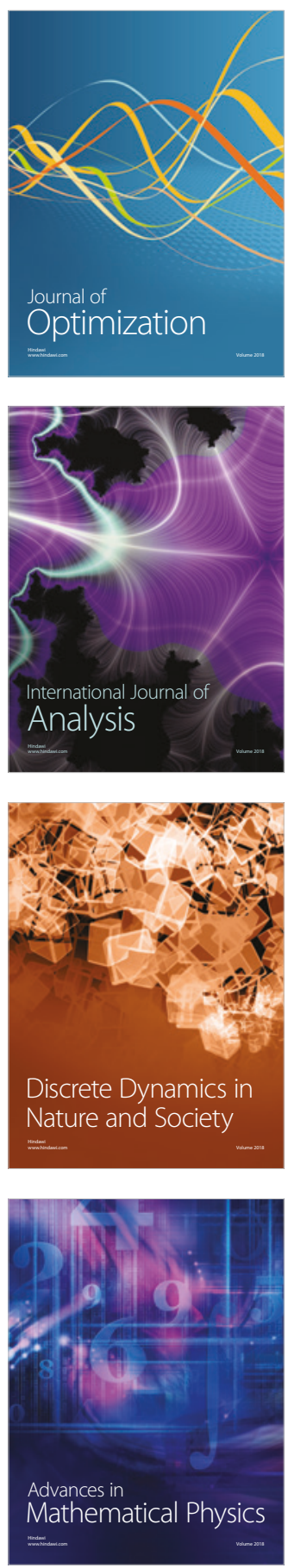